\title{
Studying twist-2 GPDs through quasi-distributions in a scalar diquark model
}

\author{
Shohini Bhattacharya* \\ Department of Physics, SERC, Temple University, Philadelphia, PA 19122, USA \\ E-mail: tug23108@temple.edu
}

\section{Christopher Cocuzza}

Department of Physics, SERC, Temple University, Philadelphia, PA 19122, USA

E-mail: tug83224 atemple.edu

\section{Andreas Metz}

Department of Physics, SERC, Temple University, Philadelphia, PA 19122, USA

E-mail: metza@temple.edu

\begin{abstract}
Studying light-cone (standard) parton distribution functions (PDFs) through Euclidean correlators in lattice QCD is currently a very active field of research. In particular, the parton quasidistributions (quasi-PDFs) suggested by Ji have attracted a lot of attention. Quasi-PDFs converge to their respective standard distributions in the limit of infinite hadron momentum. We explore the quasi-distribution approach for twist-2 generalized parton distributions (GPDs) in a frequentlyused diquark spectator model. Our analytical expressions of the quasi-GPDs reduce to their corresponding standard ones in the large-momentum limit, substantiating them to be practical tools to predict features of standard GPDs. We illustrate numerical results of quasi-GPDs and of quasiPDFs. Our focus is to test how well the quasi-distributions agree with their standard counterparts for finite hadron momenta. By discussing the sensitivity of our results to model parameters, we highlight robust features of the quasi-GPDs and quasi-PDFs that one may extract from this model study. We also discuss moments of quasi-distributions which recently attracted a lot of attention. Our contribution is based on published work and new results.
\end{abstract}

XXVII International Workshop on Deep-Inelastic Scattering and Related Subjects - DIS2019

8-12 April, 2019

Torino, Italy

\footnotetext{
* Speaker.

$\dagger$ This work has been supported by the National Science Foundation under grant number PHY-1812359, and by the U.S. Department of Energy, Office of Science, Office of Nuclear Physics, within the framework of the TMD Topical Collaboration.
} 


\section{Introduction}

Quasi parton distributions (quasi-PDFs) put forward by Ji [1] are at the forefront of numerical calculation of the partonic structure of strongly interacting systems in lattice QCD. They are defined through purely spatial correlation functions and are thus readily calculable on lattices. Quasi-PDFs approach their corresponding light-cone counterparts (standard PDFs) in the limit that the hadron momentum goes to infinity. Here we investigate this new approach to calculate (eight) twist-2 GPDs in a scalar diquark model (SDM) [2,3]. In this short write-up of the talk, we focus on the unpolarized quasi-GPD $H_{\mathrm{Q}}$ corresponding to the standard GPD $H$. All the features discussed subsequently are robust and not specific to this distribution function.

\section{Definition of Quasi-GPDs}

Analogous to the standard GPDs [4], quasi-GPDs are defined through an equal-time spatial correlation function [1],

$$
F_{\mathrm{Q}}^{[\Gamma]}\left(x, \Delta ; \lambda, \lambda^{\prime} ; P^{3}\right)=\left.\frac{1}{2} \int \frac{d z^{3}}{2 \pi} e^{i k \cdot z}\left\langle p^{\prime}, \lambda^{\prime}\left|\bar{\psi}\left(-\frac{z}{2}\right) \Gamma \mathscr{W}_{\mathrm{Q}}\left(-\frac{z}{2}, \frac{z}{2}\right) \psi\left(\frac{z}{2}\right)\right| p, \lambda\right\rangle\right|_{z^{0}=0, \vec{z}_{\perp}=\overrightarrow{0}_{\perp}},
$$

where $\mathscr{W}_{\mathrm{Q}}$ denotes a Wilson line. The unpolarized quasi-GPDs $H_{\mathrm{Q}(0 / 3)}$ and $E_{\mathrm{Q}(0 / 3)}$ are defined through the choice $\Gamma=\gamma^{0 / 3}$, the longitudinally polarized quasi-GPDs $\widetilde{H}_{\mathrm{Q}(0 / 3)}$ and $\widetilde{E}_{\mathrm{Q}(0 / 3)}$ through $\Gamma=\gamma^{0 / 3} \gamma_{5}$, and the transversely polarized quasi-GPDs $H_{T, \mathrm{Q}(0 / 3)}, E_{T, \mathrm{Q}(0 / 3)}, \widetilde{H}_{T, \mathrm{Q}(0 / 3)}$, and $\widetilde{E}_{T, \mathrm{Q}(0 / 3)}$ through $\Gamma=i \sigma^{j 0 / 3} \gamma_{5}$. See Ref. [3] for more details on the definition of quasi-GPDs.

Quasi-GPDs are functions of four kinematical variables: $x=\frac{k^{3}}{P^{3}}, \xi, t$ (or $\left|\vec{\Delta}_{\perp}\right|$ ) and $P^{3}$ (average 3-momentum of hadron). Note that the momentum fraction $x$ differs from $\frac{k^{+}}{P^{+}}$that appears for standard GPDs. Unlike the standard GPDs which extend from $-1<x<1$, the support for the quasi-GPDs is given by $-\infty<x<\infty$. We also use the quantity $\delta=\sqrt{1+\frac{M^{2}-t / 4}{\left(P^{3}\right)^{2}}}$ which shows up in the relation $P^{0}=\delta P^{3}$.

\section{Analytical Results of Quasi-GPDs in Scalar Diquark Model}

The quasi-GPD correlator in the SDM reads

$$
F_{\mathrm{Q}}^{[\Gamma]}\left(x, \Delta ; \lambda, \lambda^{\prime} ; P^{3}\right)=\frac{i g^{2}}{2(2 \pi)^{4}} \int d k^{0} d^{2} \vec{k}_{\perp} \frac{\bar{u}\left(p^{\prime}, \lambda^{\prime}\right)\left(k+\frac{\Delta}{2}+m_{q}\right) \Gamma\left(k-\frac{\Delta}{2}+m_{q}\right) u(p, \lambda)}{D_{\mathrm{GPD}}}
$$

where $D_{\mathrm{GPD}}=\left[\left(k+\frac{\Delta}{2}\right)^{2}-m_{q}^{2}+i \varepsilon\right]\left[\left(k-\frac{\Delta}{2}\right)^{2}-m_{q}^{2}+i \varepsilon\right]\left[(P-k)^{2}-m_{s}^{2}+i \varepsilon\right]$. We quote here the example of the unpolarized quasi-GPD $H_{\mathrm{Q}}$, defined with $\Gamma=\gamma^{0}$. By using Gordon identities and before carrying out the $\int d k^{0}$ one obtains

$$
H_{\mathrm{Q}(0)}\left(x, \xi, t ; P^{3}\right)=\frac{i g^{2} P^{3}}{(2 \pi)^{4}} \int d k^{0} d^{2} \vec{k}_{\perp} \frac{N_{H(0)}}{D_{\mathrm{GPD}}},
$$


where the numerator reads

$$
\begin{aligned}
N_{H(0)}= & \delta\left(k^{0}\right)^{2}-\frac{2}{P^{3}}\left[x\left(P^{3}\right)^{2}-m_{q} M-x \frac{t}{4}-\frac{1}{2} \delta \xi t \frac{\vec{k}_{\perp} \cdot \vec{\Delta}_{\perp}}{\vec{\Delta}_{\perp}^{2}}\right] k^{0} \\
& +\delta\left[x^{2}\left(P^{3}\right)^{2}+\vec{k}_{\perp}^{2}+m_{q}^{2}+(1-2 x) \frac{t}{4}-\delta \xi t \frac{\vec{k}_{\perp} \cdot \vec{\Delta}_{\perp}}{\vec{\Delta}_{\perp}^{2}}\right] .
\end{aligned}
$$

While performing $\int d k^{0}$ via contour integration, one can verify that the position of the $k^{0}$-poles never switch half planes. One therefore has the same functional form for the quasi-GPDs for any $x$, which implies that all quasi-GPDs and their derivatives are continuous functions of $x$, unlike the standard GPDs which have discontinuous derivatives at the cross-over points $x= \pm \xi$ in the SDM. (See Ref. [3] for results of all the quasi-GPDs corresponding to the twist-2 standard GPDs.)

\section{Numerical Results in Scalar Diquark Model}

Details regarding the choice of model parameters can be found in Ref. [2]. Our results discussed below are largely insensitive to variations of the model parameters [2,3]. Since most of the features exhibited by the quasi-GPDs become easier to grasp once one understands the qualitative behavior of the quasi-PDFs, we begin with discussing the PDFs first.

\subsection{Results for Quasi-PDFs}
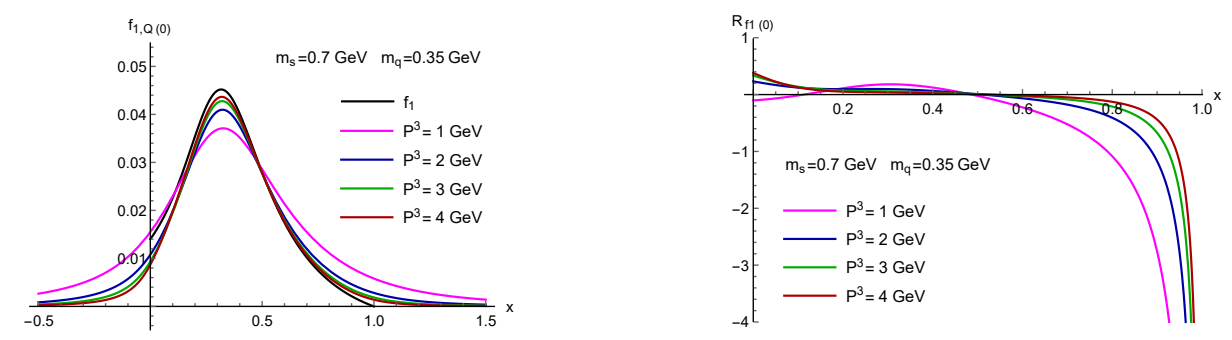

Figure 1: Left panel: Quasi-PDF $f_{1, \mathrm{Q}(0)}$ as a function of $x$ for different values of $P^{3}$. Black curve represents the standard PDF $f_{1}$. Right panel: Deviations at large $x$ made clearer through a relative-difference plot between $f_{1, \mathrm{Q}(0)}$ and $f_{1}$ as a function of $x$ for different values of $P^{3}$.

The left plot in Fig. 1 shows the unpolarized quasi-PDF $f_{1, \mathrm{Q}(0)}$, which is the forward limit of $H_{\mathrm{Q}(0)}$. One observes that for larger values of $P^{3}$, there is a good agreement between quasi and standard PDF over a wide range of $x$. However, considerable discrepancies appear as $x \rightarrow 0$ and $x \rightarrow 1$. As pointed out in [2], the discrepancy at small $x$ can be expected since, contrary to the continuous nature of the quasi-PDFs, the standard PDFs are discontinuous at $x=0$ in the SDM. The relative difference, defined as $R_{f 1(0)}\left(x ; P^{3}\right)=\frac{f_{1}(x)-f_{1, Q(0)}\left(x ; P^{3}\right)}{f_{1}(x)}$, better illustrates the discrepancies at large $x$ and is shown in the right panel of Fig.1. At $P^{3}=2 \mathrm{GeV}$, for instance, one can hardly go above $x=0.8$ for the relative difference to stay below $50 \%$.

We repeat that the two momentum fractions $\frac{k^{3}}{P^{3}}$ and $\frac{k^{+}}{P^{+}}$are different and that they cannot be related in a model-independent way. (For the rest of this sub-section we denote the former by $\tilde{x}$.) 

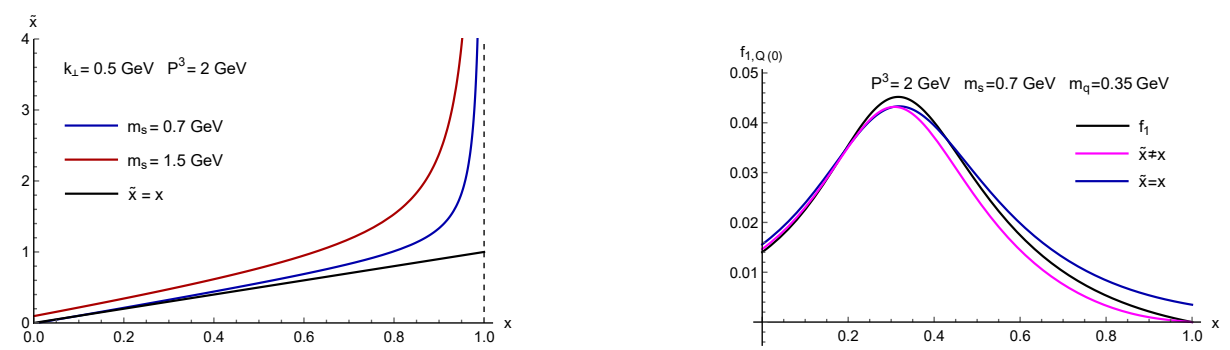

Figure 2: Left panel: Momentum fraction $\tilde{x}$ as a function of $x$ as given in Eq. (4.1) in cut-graph approach, for different values of $m_{s}$. Right panel: Impact of difference between $x$ and $\tilde{x}$ as given in Eq. (4.1) in cut-graph approach for $f_{1, \mathrm{Q}(0)}$. Note that the curves for $\tilde{x} \neq x$ go to 0 for $x \rightarrow 1$, like the standard distributions do.

However, by considering the cut-graph approximation in the SDM, one arrives at the relation

$$
\tilde{x}=x+\frac{1}{4\left(P^{3}\right)^{2}}\left(\frac{\vec{k}_{\perp}^{2}+m_{s}^{2}}{1-x}-(1-x) M^{2}\right)+\mathscr{O}\left(\frac{1}{\left(P^{3}\right)^{4}}\right) .
$$

Eq. (4.1) shows that the difference between $\tilde{x}$ and $x$ is a higher-twist effect. But most importantly, due to the $1 /(1-x)$ factor, one finds that $\tilde{x} \rightarrow \infty$ as $x \rightarrow 1$, which implies very large differences between the two momentum fractions at large $x$. This fact is highlighted in the left plot of Fig. 2. One can therefore speculate that the considerable discrepancies between the quasi-distributions and the corresponding standard distributions at large $x$ are mostly caused by the (huge) discrepancy between $\tilde{x}$ and $x$. The plot on the right panel of Fig. 2 shows that by accounting for such a highertwist "correction" in the cut-graph analysis, $f_{1, \mathrm{Q}(0)}$ indeed provides, at (very) large $x$, a better agreement with the standard PDF.

\subsection{Results for Quasi-GPDs}
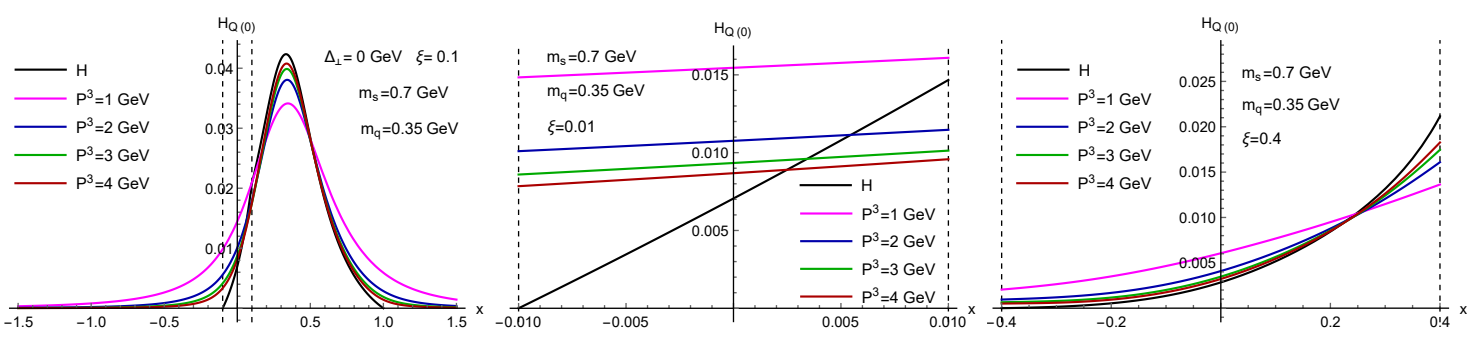

Figure 3: Left plot: Quasi-GPD $H_{\mathrm{Q}(0)}$ as a function of $x$ for $\xi=0.1$ and different values of $P^{3}$. Black curve represents the standard GPD $H$. The limits of the ERBL region are indicated by vertical dashed lines. Middle plot: $H_{\mathrm{Q}(0)}$ as a function of $x$ in the ERBL region for different values of $P^{3}$ and for $\xi=0.01$. Right plot: Result of $H_{\mathrm{Q}(0)}$ in the ERBL region for $\xi=0.4$.

The left plot in Fig. 3 shows the unpolarized $H_{\mathrm{Q}(0)}$ for $\xi=0.1$. For the skewness variable we have explored the range $0.01 \leq \xi \leq 0.4$, and below we briefly comment on the $\xi$-dependence. The convergence problem at large $x$ persists for all the quasi-GPDs whether or not they have a forward counterpart. In general, there is a tendency of the discrepancies at large $x$ to increase when $\xi$ gets larger. The significance of this feature depends on the GPD under consideration, and it is 


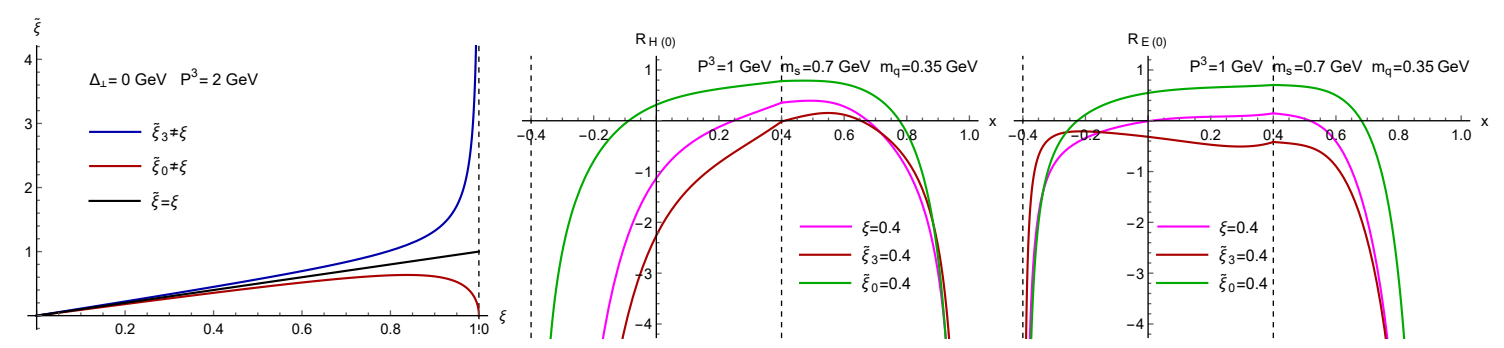

Figure 4: Left plot: Comparison of the skewness variables $\xi, \tilde{\xi}_{3}$ and $\tilde{\xi}_{0}$ for $P^{3}=2 \mathrm{GeV}$ and $\left|\vec{\Delta}_{\perp}\right|=0 \mathrm{GeV}$. Middle plot: Majority's trend reflected through a relative-difference plot between $H_{\mathrm{Q}(0)}$ and $H$ for three different definitions of the skewness variable. Right plot: Outlier's trend reflected through a relative-difference plot between $E_{\mathrm{Q}(0)}$ and $E$.

most pronounced for the quasi-GPDs $\widetilde{E}_{\mathrm{Q}}$ and $\widetilde{E}_{T, \mathrm{Q}}$. The middle and right plots in Fig. 3 shows $H_{\mathrm{Q}(0)}$ for just the ERBL region for $\xi=0.01$ and $\xi=0.4$ respectively. Generally, for small $\xi$ one finds significant deviations between the quasi-GPDs and the corresponding standard GPDs. This situation is the GPD counterpart of the problem for quasi-PDFs around $x=0$. For small $\xi$, the standard GPDs rapidly approach zero at $x=-\xi$ in a very narrow $x$-range, whereas the quasiGPDs are much smoother in that range. Once $\xi$ is increased, we observe a (much) better agreement between quasi-GPDs and the standard GPDs for a large fraction of the ERBL region. This outcome suggests that lattice calculations could provide very valuable information in the ERBL region, provided that the skewness is not too small.

So far we have used the same skewness variable $\xi$ for both the standard GPDs and the quasiGPDs. However, for the quasi-GPDs one could in principle consider different variables to describe the longitudinal momentum transfer to the hadron. Examples in this context are $\tilde{\xi}_{3}=-\frac{\Delta^{3}}{2 P^{3}}$ and $\tilde{\xi}_{0}=-\frac{\Delta^{0}}{2 P^{0}}$. These "quasi skewness" variables are related to the standard skewness via a highertwist effect encoded in the model-independent relations $\tilde{\xi}_{3}=\delta \xi$ and $\tilde{\xi}_{0}=\frac{\xi}{\delta}$. The left plot of Fig. 4 shows considerable differences between $\xi, \tilde{\xi}_{3}$, and $\tilde{\xi}_{0}$ when $P^{3}$ is small. We explored the impact of the difference between these variables on the quasi-GPDs. Through the specific example of $H_{\mathrm{Q}(0)}$, the middle plot of Fig. 4 shows that ignoring the higher-twist effect and using $\tilde{\xi}_{3}$ leads to a better convergence of the majority of the quasi-GPDs for most of the DGLAP region. The only outliers in that regard are $E_{\mathrm{Q}(0)}, \widetilde{E}_{\mathrm{Q}(0 / 3)}$ and $E_{T, \mathrm{Q}(0)}$, where $E_{\mathrm{Q}(0)}$ is shown as a representative case in the right plot of Fig. 4. Also, using the variable $\tilde{\xi}_{0}$ typically gives poorer convergence for the quasi-GPDs. Our conclusions also hold for even larger values of $\xi$, where the numerical discrepancy between the three skewness variables increases further — see left plot of Fig. 4.

\section{Moments}

We first consider the lowest moment of the quasi-GPD $H_{\mathrm{Q}}$. Including a flavor index ' $q$ ' one finds the model-independent relation

$$
\int_{-1}^{1} d x H^{q}(x, \xi, t)=\int_{-\infty}^{\infty} d x \frac{1}{\delta} H_{\mathrm{Q}(0)}^{q}\left(x, \xi, t ; P^{3}\right)=\int_{-\infty}^{\infty} d x H_{\mathrm{Q}(3)}^{q}\left(x, \xi, t ; P^{3}\right)=F_{1}^{q}(t),
$$


where $F_{1}$ is the well-known Dirac form factor. The lowest moment of standard GPDs depends on $t$, but does not depend on $\xi$. The quasi-GPDs also depend on $P^{3}$, but remarkably this dependence also drops out in the lowest moment. However one must divide half of the quasi-GPDs by the kinematical factor $\delta$ in order to arrive at this result. Since $\delta$ describes a higher-twist effect, including this factor is in principle a matter of taste. But the moment analysis suggests that taking into account $\delta$ like in (5.1) appears natural.

We now turn our attention to the second moment of quasi-GPDs considering again only the vector operator $\bar{\psi}^{q} \gamma^{\mu} \psi^{q}$. In close analogy to the celebrated expression for the second moment of $H+E$, namely $\int_{-1}^{1} d x x\left(H^{q}(x, \xi, t)+E^{q}(x, \xi, t)\right)=A^{q}(t)+B^{q}(t)$ where $A^{q}(0)+B^{q}(0)=J^{q}$ is the total angular momentum for the quark flavor ' $q$ ', one then finds for the quasi-GPDs

$$
\begin{aligned}
\int_{-\infty}^{\infty} d x x \frac{1}{\delta}\left(H_{\mathrm{Q}(0)}^{q}\left(x, \xi, t ; P^{3}\right)+E_{\mathrm{Q}(0)}^{q}\left(x, \xi, t ; P^{3}\right)\right)= & \frac{1}{2}\left(\delta^{2}+1\right)\left(A^{q}(t)+B^{q}(t)\right) \\
& +\frac{1}{2}\left(\delta^{2}-1\right) D^{q}(t), \\
\int_{-\infty}^{\infty} d x x\left(H_{\mathrm{Q}(3)}^{q}\left(x, \xi, t ; P^{3}\right)+E_{\mathrm{Q}(3)}^{q}\left(x, \xi, t ; P^{3}\right)\right)= & A^{q}(t)+B^{q}(t) .
\end{aligned}
$$

Note that in Eq. (5.2) the form factor $D^{q}$ of the anti-symmetric part of the EMT enters. One can conclude that the second moment of $H_{\mathrm{Q}(3)}+E_{\mathrm{Q}(3)}$ is directly related to the angular momentum of quarks, while for $H_{\mathrm{Q}(0)}+E_{\mathrm{Q}(0)}$ this relation contains a higher-twist "contamination." The modelindependent expressions for the moments of the quasi distributions are potentially significant as they may be useful for studying the systematic uncertainties of results from lattice QCD, especially due to the fact that the $P^{3}$-dependence of the moments is either computable or nonexistent.

\section{Summary}

We have studied twist-2 GPDs through parton quasi-distributions in the SDM. Our analytical expressions for the quasi-GPDs reduce to the respective standard GPDs for $P^{3} \rightarrow \infty$ further validating that quasi-GPDs could be a viable tool for getting information about standard GPDs. For finite $P^{3}$ and large $\xi$, quasi-GPDs agree well with the standard distributions in the ERBL region. This agreement gets poorer if $\xi$ gets smaller. We have provided a model-independent analysis of moments of quasi-GPDs including the relation to Ji's spin-sum rule. The moment analysis may assist in the study of systematic uncertainties in lattice QCD.

\section{References}

[1] X. Ji, Parton physics on a Euclidean lattice, Phys. Rev. Lett. 110, 262002 (2013).

[2] S. Bhattacharya, C. Cocuzza and A. Metz, Generalized quasi parton distributions in a diquark spectator model, Phys. Lett. B 788, 453 (2019).

[3] S. Bhattacharya, C. Cocuzza and A. Metz, Exploring twist-2 GPDs through quasi-distributions in a diquark spectator model, arXiv:1903.05721 [hep-ph].

[4] M. Diehl, Generalized parton distributions, Phys. Rept. 388, 41 (2003). 